possible variations highly suggestive and that I, having contributed to the standard model which gives no hint of an explanation of such variations, should find the data statistically unconvincing.

With new detectors to measure the energy spectrum and precise time dependence of solar neutrinos, and terrestrial global networks, as well as polar observations and space experiments to determine more precisely the spectrum of pressure oscillations, solar physics is in a renaissance period. Similar techniques have produced indications of oscillations on the surfaces of other stars and many are optimistic that definitive measurements will be forthcoming shortly. To date, the most surprising thing is that not much has been shown to be definitely new under the Sun. Surely, new data will lead soon to big surprises concerning the interiors of stars. Won't they?

John Bahcall is at the Institute for Advanced Study, Princeton, New Jersey 08540, USA.

1. Hirata, K.S. et al. Phys. Rev. Lett. 63, 16-19 (1989).

. Korzennic, S.G. \& Uirich, R.K. Astrophys. J. 339, 1144 1155 (1989).

3. Christensen-Dalsgaard, J. et al. Nature 315, 378-382 (1985).

\title{
Spinning ties that bind
}

\section{Paul Calvert}

SPIDERS make five different kinds of silk for making the web scaffolding, the sticky capture spiral, the egg sac, the attachment disks and wrapping bands for prey. Each type of silk seems to come from a specific spinning gland, although it is actually rather hard to tell which spinneret a spider is using at any one time ${ }^{1}$. Amino-acid analyses show that there are differences among the various silks, but composition is not a good guide to polymer properties and so says little about why the silks have different functions. On page 305 of this issue $^{2}$, Vollrath and Edmonds discuss the origin of the elasticity of the capture spiral when compared with the stiffness of the radial frame threads.

The frame thread is a single filament of about $1 \mu \mathrm{m}$ thick, whereas the capture thread is a pair of filaments of $0.7 \mu \mathrm{m}$ thick with a coating of sticky (viscid) fluid. The capture thread must have many of the properties of chewing gum. It must be adhesive, it must extend to very large strains without breaking, and it must have a high tensile strength. A strong but stiff net does not spread the load well, so threads can be broken one at a time by a determined captive. As was pointed out by Gordon $^{3}$, many biological materials show a J-shaped stress-strain curve. The initial modulus is very low, but the material becomes progressively difficult to extend so that the stress rises rapidly before breaking occurs. Skin is one example of this combination of softness and strength. Vollrath and Edmonds ${ }^{2}$ show a similar curve for the catching spiral in their Fig. $2 a$ on page 306 , where the ' $\mathrm{J}$ ' has a very long tail before rising at 3 times the original length.

This long tail is achieved by the coiling of the capture thread within droplets of the coating fluid. A long, thin thread of liquid is unstable (the Rayleigh instability) and should break up into a series of drops. This happens to a thin stream of water flowing from a tap. As seen in Vollrath and Edmonds' Fig. 1 on page 306, the thread is apparently well wetted by the coating and so coils up inside the drops until it is taut in the frame. The initial large extension thus results only from uncoiling of the thread.

In the next stage of extension, the thread itself is stretched. The central argument of Vollrath and Edmonds concerns why this thread is softer then the frame thread. They suggest that the capture thread is plasticized by water from the viscid layer which surrounds it. The alternative would be to argue for a difference in structure or composition.

It is very hard to estimate the moduli of fibres because it is hard to measure their diameter. From the data presented by Vollrath and Edmonds, the modulus of the capture thread is in the range of $1-5 \times 10^{7}$ pascal when wet and about ten times this when dry, whereas the dry frame thread is Beauty from strength. The silk filaments of the orb weaver's web catch the morning dew. (Courtesy of Bruce Coleman Limited.) comparison, Nylon, which is close in composition to silk, has a modulus of $10^{\circ}$ pascal as spun and $5 \times 10^{9}$ pascal when it has been drawn (stretched) into a textile fibre with its molecular chains oriented parallel to the axis. Like Nylon, silk does soften in a humid environment. Water breaks up the hydrogen bonding between chains and so reduces the modulus. Gosline and coworkers ${ }^{4}$ have shown that this effect is large in spider dragline silk (which is similar to the web frame). Exposure to water reduces the modulus from $10^{10}$ to $10^{7}$ pascal. As with most textile fibres, the silk structure is a blend of crystalline and amorphous regions. The water has little effect on the crystals but converts the hard amorphous regions into a soft rubber. The water weakens, but does not eliminate, the inter-chain bonding, and the material shows viscoelasticity (slow recovery after stretching). A similar effect is seen in cold rubber. Vollrath and Edmonds go on to show that the viscoelastic responses of wet and dry frame and spiral silks are similar.

We now have a number of possibilities. First, the core of the capture thread is the same as the frame, but kept moist by the viscid coating. This could not last for a long time as the thread is very fine and must dry rapidly. Spiders spin their webs just before dawn and so they may well lose efficiency as the air dries out. In this case, the composition of the thread would be the same as for the frame. It would help to know whether the frame and spiral core came from the same spinneret but this seems to be uncertain ${ }^{1}$.

Second, the capture thread has a different composition, which makes it more hydrophilic and so more able to retain water and be plasticized when the frame thread dries. Gosline et al. found that the dragline thread is stiff at $20^{\circ} \mathrm{C}$ and 50 per cent humidity.

Third, the capture thread has a more irregular structure which makes it less crystalline. The greater amorphous content would make the thread more rubbery. This could be achieved with little or no change in amino acid composition. And finally, the capture thread is the same composition as the frame but is spun into a state of lower orientation.

Vollrath and Edmonds argue for the first case. It would be nice to know more, but funding in this area is not abundant. The way to resolve the problem may be to find a major application for webs perhaps a missile defence system?

Paul Calvert is in the Materials Laboratories at the University of Arizona, Tucson, Arizona 85712, USA.

1. Witt, P.N., Reed, C.F. \& Peakall, D.B. A Spider's Web. Problems in Regulatory Biology (Springer, New York, 1968).

2. Vollrath, F. \& Edmonds, T. Nature 340, 305-307 (1989),

3. Gordon, J.E. Structures, Or Why Things Don't Fall Down (Penguin, Harmondsworth, 1978).

4. Gosline, J.M., Denny, M.W. \& DeMont, M.E. Nature 309 , 551-552 (1984). 